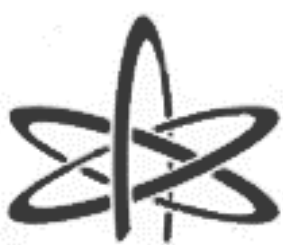

BJRS
BRAZILIAN JOURNAL

$\mathrm{OF}$

RADIATION SCIENCES

06-03 (2018) 01-24

\title{
Estudo dos principais indicadores edafológicos da transferência solo-planta para o iodo
}

\author{
F. Bartoly ${ }^{a}$, M.A.V. Wasserman ${ }^{b}$, M.M. da Silva ${ }^{a}$, P.A. de Lima ${ }^{b}$ \\ ${ }^{a}$ Programa de Pós-Graduação em Radioproteção e Dosimetria /Instituto de Radioproteção e Dosimetria. \\ CEP 22783-127, Rio de Janeiro - RJ, Brasil \\ ${ }^{b}$ SETER/Instituto de Engenharia Nuclear. CEP 21941-972, Rio de Janeiro, RJ, Brasil \\ mwasserman@ien.gov.br
}

\section{RESUMO}

Os modelos radioecológicos computacionais utilizados para fins de segurança ambiental dependem de valores de parâmetros nem sempre disponíveis com a especificidade ambiental requerida. Dentro do cenário agrícola, o fator de transferência solo-planta $(F v)$ é uma informação crucial para a avaliação do risco radiológico devido à ingestão de alimentos. A transferência solo-planta está diretamente relacionada com a fenologia e fisiologia das plantas, propriedades dos solos e propriedades físico-químicas dos radionuclídeos. Este trabalho teve por objetivo identificar, através da revisão sistemática da literatura, as propriedades dos solos que afetam os valores de $F v$ para o iodo, e, a partir dessas, propor indicadores edafológicos potencialmente capazes de prever valores de $F v$ através do uso de ferramentas de inteligência artificial. A análise da literatura permitiu selecionar o teor de matéria orgânica e o pH dos solos como potenciais indicadores edafológicos de valores de $F v$. Apesar do número considerável de artigos publicados sobre o tema, foram levantados e estruturados em um banco de dados somente 135 valores de $F v$ para o iodo, que puderam ser associados à pelo menos uma propriedade do solo. Desse banco de dados, somente 2 conjuntos de aprendizado ("learning sets") puderam ser estruturados para a o treinamento de uma rede neural artificial, e somente para o ${ }^{125} \mathrm{I}$ : um para trigo $(n=38)$ e outro para alface $(n=14)$. Esse resultado aponta que os registros de valores $F v$ para o iodo, não reportam as informações ambientais adequadas para a análise desse parâmetro, sendo que a maioria das informações disponíveis na literatura foram restritas a solos de clima temperado.

Palavras-chave: matéria orgânica, iodo, $\mathrm{pH}$

Accepted for publication 2018-09-03 


\begin{abstract}
Computational radioecological models used for environmental safety purposes depend on parameter values not always available with the required environmental specificity. Within the agricultural scenario, the soil-plant transfer factor $(F v)$ is a crucial information for the evaluation of the radiological risk due to the ingestion of food. The soil-plant transfer is directly related to plant phenology and physiology, soil properties and physicochemical properties of radionuclides. The objective of this work was to identify, through the systematic review of the literature, the soil properties that affect iodine Fv values and, from these, to propose its potential soil indicators to forecast Fv values by artificial intelligence tools. The literature review allowed to select the organic matter content and the $\mathrm{pH}$ of the soils as potential soil indicators of Fv values. Despite the abundant literature on the subject, only $135 F v$ values for iodine could be associated with at least one soil property that were collected and structured in a database. From this database, only 2 learning sets were structured for training of an artificial neural network and, only for ${ }^{125} \mathrm{I}$ : one for wheat $(\mathrm{n}=38)$ and one for lettuce $(\mathrm{n}=14)$. This result indicates that the records of $\mathrm{FV}$ values do not report the adequate environmental information for the analysis of this parameter, and all information was restricted to temperate or subtropical climate soils.
\end{abstract}

Key words: organic matter, iodine, $\mathrm{pH}$. 


\section{INTRODUÇÃO}

A transferência de radionuclídeos do solo para as plantas determina a extensão da contaminação e, consequentemente, o risco de exposição radioativa da população devido à ingestão de alimentos. Os modelos radioecológicos computacionais utilizados para fins de segurança ambiental dependem de dados nem sempre disponíveis com a especificidade ambiental requerida. Dentro do cenário agrícola, o fator de transferência solo-planta $(F v)$ é uma informação crucial para a avaliação do risco radiológico devido à ingestão de alimentos e constitui um bom exemplo em sua especificidade ambiental . O $F v$ relaciona a concentração do radionuclídeo na parte comestível da planta ( $\mathrm{Bq} \mathrm{kg} \mathrm{kg}^{-1}$ em peso seco), com a concentração presente no solo $\left(\mathrm{Bq} \mathrm{kg}^{-1}\right.$ em peso seco) . A transferência solo-planta está diretamente relacionada com a fenologia e fisiologia das plantas, propriedades dos solos e propriedades físico-químicas dos radionuclídeos. Assim, diferentes tipos de solos, plantas e níveis de tecnologias envolvidas na produção agrícola, resultam em uma grande dispersão de valores $F v$ medidos para um dado radionuclídeo. Dependendo das propriedades do solo e do tempo decorrido após a contaminação, um mesmo radionuclídeo pode apresentar valores de $F v$ com até 5 ordens de magnitude de diferença para um mesmo vegetal (ou grupo de vegetais), como já observado para o ${ }^{137} \mathrm{Cs}$ em cereais . Estudos radioecológicos realizados, relativos ao $F v$ de radionuclídeos $\left({ }^{137} \mathrm{Cs} \mathrm{e}{ }^{90} \mathrm{Sr}\right)$, apontam que parâmetros pedológicos explicam consideravelmente o comportamento dos mesmos no sistema solo-planta. Foi observado que valores de $F v$ para ${ }^{137} \mathrm{Cs}$ determinados em solos brasileiros, podem ser duas ordens de grandeza superiores aos valores de $F v$ para cereais, medidos em solos europeus afetados pelo acidente de Chernobyl. Desse modo, o uso de valores $F v$ genéricos ou valores de referência propostos pela IAEA, em substituição à valores regionais, pode subestimar ou superestimar riscos. No entanto, a extensão e amplitude de ação das propriedades dos solos ainda permanecem desconhecidos para muitos radionuclídeos .

Nas primeiras semanas após o acidente de Chernobyl, o ${ }^{131} \mathrm{I}$ foi o principal contribuinte de dose para a população na Bielorrússia, Rússia e Ucrânia em função da sua elevada concentração 
no leite consumido . O mais recente acidente nuclear de Fukushima (2011) também promoveu a liberação de iodo nos ecossistemas [8].

Além das liberações acidentais proveniente de reatores nucleares, o isótopo ${ }^{129} \mathrm{I}$ também pode ser encontrado no meio ambiente oriundo dos testes nucleares e por atividades industriais rotineiras, como as instalações de reprocessamento do combustível nuclear. Esta grande quantidade de ${ }^{129}$ I liberado no meio ambiente pela atividade nuclear humana, promoveu uma elevação da relação ${ }^{129} \mathrm{I} /{ }^{127} \mathrm{I}$ em pelo menos duas ordens de grandeza em comparação com os valores naturais ;. Muito dos radionuclídeos liberados no meio ambiente em função destes testes, podem ser encontrados nos solos e nos alimentos de todo o mundo. Atualmente, o reprocessamento do combustível nuclear é a principal fonte do ${ }^{129} \mathrm{I}$ artificial no meio ambiente.

O iodo é um elemento essencial para o corpo humano e para os animais . É conhecido por ser concentrado na glândula tireoide, e sua presença dentro do organismo é necessária para a síntese de hormônios responsáveis pelo controle do metabolismo e o seu equilíbrio . Portanto, do ponto de vista da radioproteção, o conhecimento do comportamento deste elemento no meio ambiente, principalmente no ecossistema terrestre é imprescindível para avaliar doses.

Este trabalho teve por objetivo identificar, através da revisão sistemática da literatura, as propriedades dos solos que afetam os valores de $F v$ para o iodo e, a partir dessas, propor indicadores edafológicos potencialmente capazes de prever valores de $F v$, através do uso de ferramentas de inteligência artificial.

\section{MATERIAIS E MÉTODOS}

Denomina-se revisão sistemática da literatura, a revisão planejada por métodos sistemáticos, que visa identificar, selecionar e avaliar criticamente estudos relevantes sobre uma questão claramente formulada . Para o processo de revisão sistemática deste estudo, os seguintes passos foram estabelecidos: 


\subsection{Foco da Pesquisa:}

O objetivo dessa pesquisa foi levantar as propriedades dos solos que afetam o comportamento do iodo em solos e elaborar um conjunto de aprendizado ("learning set") para ferramentas de IA, através de valores de $F v$ para o iodo disponíveis na literatura.

\subsection{Processo de busca:}

Para o foco da pesquisa, foram consultadas inicialmente as seguintes Bases de Dados eletrônicas: Web of Science core collection (http://apps.webofknowledge.com; última visualização em 09/05/2018) e Periódico Capes (http://www.periodicos.capes.gov.br; última visualização em 09/05/2018). A base de dados do portal Periódico Capes, embora menos abrangente que a Web of Science, possui várias coleções relevantes para o tema, dentre elas: MEDLINE/PubMed; AGRIS (United Nations, Food and Agriculture Organization), ScienceDirect Journals (Elsevier) e SpringerLink. Foram revisadas as listas de referência e resumos disponíveis para triar estudos potencialmente relevantes. Adicionalmente, foi realizada a busca manual por referências citadas nos artigos relevantes, o que conduziu a alguns artigos anteriores ao período de busca (a partir de 1986), bem como a inclusão de alguns relatórios técnicos, em função da disponibilidade de valores de $F v$. A busca englobou os estudos mais relevantes para os focos de interesse e algumas outras abordagens ambientais genéricas e introdutórias ao estudo. A palavra chave usada para esta pesquisa no idioma em inglês foi: "iodine*soil" e o período de busca eletrônica selecionado foi após o ano de 1986.

\subsection{Critérios de inclusão e exclusão:}

Para a revisão bibliográfica, com o intuito de identificar os indicadores edafológicos do iodo, foram utilizados somente os artigos que abordavam os processos biogeoquímicos relacionados a origem, destino e comportamento do iodo no sistema solo-planta. Artigos especificamente relacionados aos processos de deposição foliar e translocação do iodo no interior da planta, avaliação de procedimentos analíticos, avaliação de dose e impactos na saúde, transferência para animais e 
alimentos de origem animal, ambientes aquáticos e processos hidrogeoquímicos, processos atmosféricos e processos geológicos foram excluídos.

Para a inclusão de valores de $F v$ no banco de dados, foram utilizados os seguintes critérios:

- Considerar valores de $F v$ provenientes de estudos in situ e estudos experimentais, desde que os procedimentos metodológicos estivessem em acordo com os critérios estabelecidos pela União Internacional de Radioecologistas (IUR), para a determinação de valores de $F v$.

- Considerar valores de $F v$ que continham pelo menos uma propriedade físico-química do solo subjacente.

Ao citar os valores das referências, foi relatado o número de dígitos significativos utilizados pelos autores originais. Os dados aceitos foram reunidos em uma planilha Excel. O banco de dados bibliográfico foi organizado em Endnote.

\section{RESULTADOS E DISCUSSÕES}

De acordo com o levantamento bibliográfico realizado, notou-se um significativo aumento do estudo do iodo no sistema solo-planta após 2002 (Figura 1).

Figura 1: Distribuição do número de publicações por ano, após o acidente de Chernobyl. 


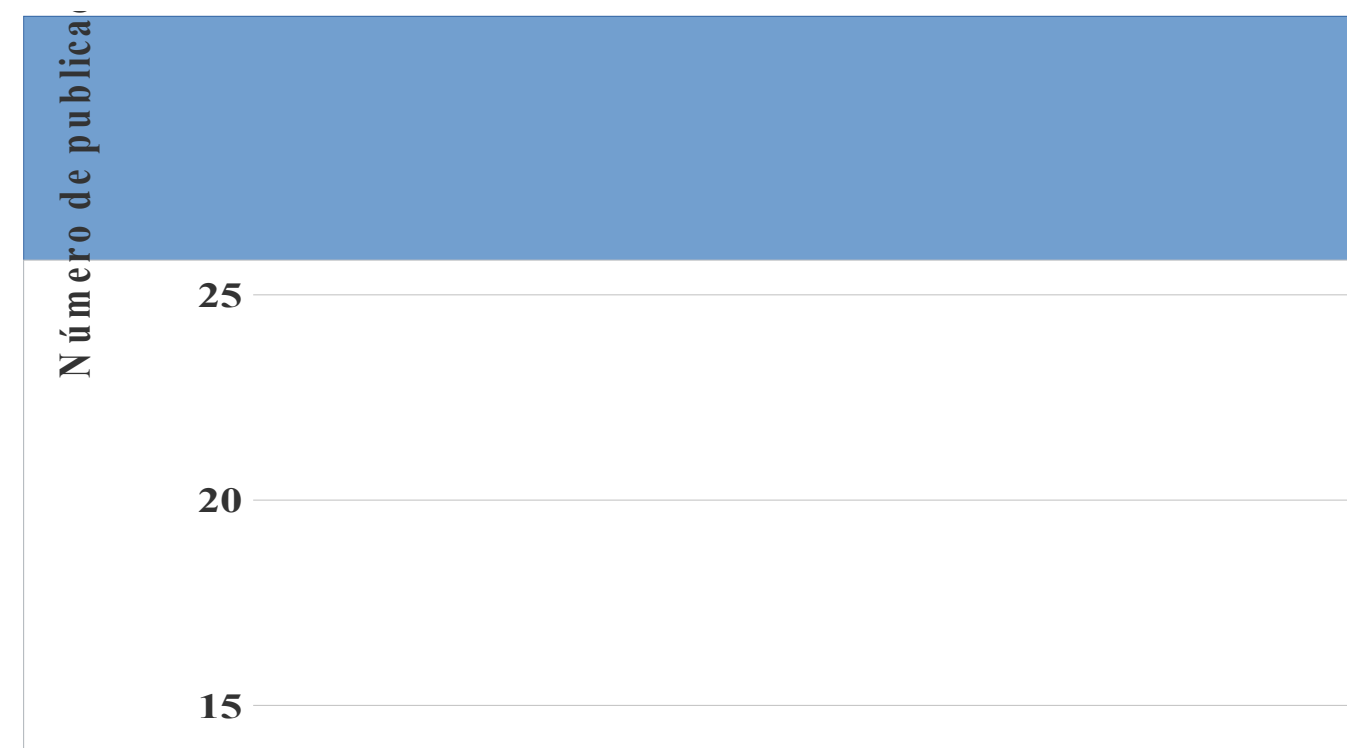

Possivelmente, a atribuição do aumento do número de casos de câncer na tireoide, observados em crianças expostas ao fallout do acidente de Chernobyl, relatado em 1992 , associado à necessidade de se avaliar o impacto ambiental do ${ }^{129} \mathrm{I}$ liberado de instalações nucleares e ao reconhecimento pela ICCIDD (International Council for the Control of Iodine Deficiency Disorders) em 1999, de que a deficiência do iodo estável na alimentação, tornou-se uma ameaça para o desenvolvimento econômico e social de muitos países, justificam o crescente interesse no comportamento deste elemento no sistema solo-planta. Os valores de $F v$ para o iodo estável $\left({ }^{127} \mathrm{I}\right)$ observados na Tabela 1, especificados em biofortificação, apontam o aumento da preocupação com o déficit da ingestão deste elemento pela população. Além disso, selecionando o número de artigos revisados com valores de $F v$ solo-planta por tipo de iodo, foi observado apenas um artigo abordando valores para o ${ }^{131} \mathrm{I}$. Provavelmente, esta análise restrita, ocorre em função da meia vida do ${ }^{131}$ I ser de aproximadamente 8 dias, e os estudos relacionados a dose por ingestão de plantas contaminadas por este tipo de iodo, estão muito mais relacionados com a deposição atmosférica foliar (transferência ar-planta). Os valores de $F v$ gerados, através de contaminação artificial com o iodo, têm como destaque, o uso do isótopo ${ }^{125} \mathrm{I}$, que tem sido usado como radiotraçador. Já os estudos com o ${ }^{129}$ I também foram bem relevantes. 
Tabela 1. Número de artigos revisados com valores de Fv solo-planta por tipo de iodo

\begin{tabular}{ccc}
\hline Tipo de iodo & Total de $\mathbf{F v}$ & Número de artigos \\
\hline${ }^{125} \mathrm{I}$ & 170 & 8 \\
${ }^{127} \mathrm{I}$ & 105 & 10 \\
${ }^{129} \mathrm{I}$ & 81 & 5 \\
${ }^{131} \mathrm{I}$ & 6 & 1 \\
I não especificado & 3 & 1 \\
\hline
\end{tabular}

Possivelmente, as altas concentrações de ${ }^{129} \mathrm{I}$ de origem antropogênica no meio ambiente, somado a uma meia vida de aproximadamente 15,7 milhões de anos, estabelece a necessidade do conhecimento do seu comportamento geoquímico e possíveis fitodisponibilidade.

A Tabela 2 apresenta os principais temas abordados nos 309 artigos selecionados. Esta distribuição reforça a predominância de estudos que geram subsídios para a compreensão dos impactos do iodo no ambiente e na saúde humana, em decorrência do reprocessamento do combustível nuclear apontados em migração e rejeitos, por exemplo; e de sua deficiência em alimentos, observados nos artigos que tratavam de biofortificação.

Tabela 2. Número de artigos revistos, distribuídos por seus principais temas abordados.

\begin{tabular}{cc}
\hline Assunto & Número de artigos \\
\hline Mecanismos de sorção & 89 \\
Absorção radicular; fator de transferência solo/planta & 35 \\
Chernobyl & 34 \\
Fukushima & 25 \\
Biofortificação & 32 \\
Migração & 28 \\
Rejeitos & 27 \\
Saúde humana & 17 \\
Toxicidade & 15 \\
Microrganismos & 11 \\
Remediação & 8 \\
Técnicas analíticas (Fora do tema) & 3 \\
\hline
\end{tabular}


As revistas que concentram mais de 1/3 das publicações são: a Journal of Environmental Radioactivity (62), Science of the Total Environment (18), Journal of Radioanalytical and Nuclear Chemistry (14) e Soil Science and Plant Nutrition (9).

\subsection{Propriedades dos solos relevantes para a compreensão da dinâmica do iodo no sistema solo-planta.}

O aumento da concentração do iodo de origem antropogênica no ambiente terrestre, seja através de acidentes em usinas nucleares, testes atômicos e devido às liberações de rotinas das indústrias de reprocessamento, têm sido responsáveis pelo aumento da concentração do iodo nos solos. No entanto, uma vez no solo, algumas propriedades demonstraram ser relevantes para explicar o comportamento do iodo, interferindo diretamente na sua fitodisponibilidade. Portanto, buscou-se na literatura, as propriedades dos solos com maior relevância para os mecanismos de retenção e mobilidade do iodo neste ambiente. A Tabela 3, apresenta as propriedades dos solos que mais se destacaram nesta revisão como relevantes para a compreensão da dinâmica do iodo no sistema solo-planta, com o seu respectivo quantitativo de artigos que abordavam o tema, dentro do período da pesquisa (após 1986), destacando-se algumas referências (últimos cinco anos). De acordo com essa Tabela, verifica-se que a matéria orgânica foi a mais representativa, e seu papel está associado como um dos principais responsáveis pela retenção do iodo nos solos. No entanto, ela não foi a única fração responsável pela dinâmica do iodo. A textura, ou seja; a quantidade de argila e coloides dos solos, assim como os óxidos e hidróxidos de Fe e Al influenciam a capacidade de adsorção do iodo nos solos. O pH o Eh e os microrganismos, influenciam nas espécies químicas do iodo presentes no solo.

Tabela 3. Propriedades dos solos referenciadas como relevantes para a compreensão da dinâmica do iodo no sistema solo-planta.

$\begin{array}{lll}\text { Propriedades } & \text { Total de artigos } & \text { Referências (últimos } 5 \text { anos) } \\ \text { relevantes do } & \text { que abordavam }\end{array}$




\begin{tabular}{|c|c|c|}
\hline solo & a propriedade & \\
\hline $\begin{array}{l}\text { Matéria } \\
\text { Orgânica }\end{array}$ & 135 & $\begin{array}{l}\not ; \\
;\end{array}$ \\
\hline $\mathrm{pH}$ & 92 & , \\
\hline Eh & 35 & ;,;;;,;;,;,;,;,;;; \\
\hline Textura & 53 & $;, ;, ;, ;, ;, ;, ;, ; ;$ \\
\hline Mineralogia & 56 & $;, ;, ;, ;, ;, ;$ \\
\hline Microrganismos & 28 & $;, ;, ; ; ;$ \\
\hline
\end{tabular}

\subsection{Avaliação dos potenciais indicadores pedológicos da fitodisponibilidade do iodo.}

\subsubsection{A Matéria Orgânica do Solo}

Muitos trabalhos existentes na literatura, evidenciam que a retenção do iodo é influenciada pelo conteúdo de matéria orgânica dos solos . Na maioria dos solos, o teor de matéria orgânica varia de 0,5 a 5,0\% nos horizontes minerais superficiais, podendo apresentar valores mais elevados em solos orgânicos . A matéria orgânica do solo (MOS) refere-se a todos os compostos que contêm carbono orgânico no solo, incluindo microrganismos vivos e mortos, resíduos de plantas e animais parcialmente decompostos, produtos de sua decomposição e substâncias orgânicas microbiologicamente e/ou quimicamente alteradas . 
Apesar de estar em pequenas quantidades, quando comparada com a fração mineral, a matéria orgânica no solo é de extrema importância para os sistemas de produção agrícola devido aos diversos efeitos que produz nas propriedades químicas, físicas e biológicas do solo e por seu efeito no crescimento e desenvolvimento das plantas. Nos perfis de solo, os níveis mais altos de iodo são frequentemente encontrados nas camadas superficiais, onde também é maior o conteúdo orgânico. A vegetação viva presente no solo, também pode reter o iodo que poderia ser carreado pelas chuvas. Posteriormente, quando esta vegetação morre, este elemento estará contido nesta matéria orgânica fresca, podendo se tornar gradativamente disponível no solo . Contudo, a decomposição desta vegetação, para formar a matéria orgânica do solo, aumenta a capacidade de fixar o iodo no solo.

Solos classificados com baixo teor de matéria orgânica, como os Podzols, por exemplo, são geralmente pobres em iodo; enquanto os Chernozems e os Solos de Turfa, com elevado teor de matéria orgânica, são ricos em iodo . Os níveis de iodo nos Solos de Turfa são mais altos nas camadas orgânicas (horizonte $\mathrm{O}$ ) e muito mais baixos nos horizontes subsequentes. Nos Podzols, o iodo irá se acumular no material orgânico presente no horizonte A; e, no horizonte B, estará associado aos óxidos de ferro e alumínio .

A boa correlação do iodo com a matéria orgânica da superfície dos solos também pode ser explicada considerando que a maior parte deste elemento no solo é originado da atmosfera e não das rochas mães. Portanto, as camadas superficiais dos solos costumam ter as maiores concentrações de iodo retido pela matéria orgânica, enquanto as camadas mais profundas, terão uma menor quantidade ou até mesmo nenhuma entrada de iodo.

O iodo no solo pode ser encontrado em várias formas químicas. Muitos estudos têm apontado, que o iodo na forma de iodeto $\left(\mathrm{I}^{-}\right)$, forma uma ligação com o material orgânico do solo . A ligação do iodo com a matéria orgânica dos solos, ocorre em pH inferior a 6,9 .

Com base nos principais estudos que abordam a influência da matéria orgânica na dinâmica do iodo, espera-se que a mesma influencie de modo significativo os valores de $F v$ medidos em um solo, e, por essa razão, será considerada uma informação crucial para a previsão desses valores para o iodo. 


\subsubsection{Os componentes minerais: textura e composição}

A capacidade de adsorção do iodo em solos também parece depender da quantidade de argila e coloides presentes . Fuge e Johnson em um trabalho de revisão sobre o iodo, apontaram que alguns autores observaram solos argilosos com altos teores deste elemento; e outros, relatando a presença de uma grande concentração de iodo, em solos ricos em óxidos e hidróxidos de ferro e alumínio. Whitehead em um trabalho de distribuição e transformação do iodo no meio ambiente, apontou os óxidos de alumínio como os mais importantes na retenção do iodo nos solos, com valores de pH acima de 4,8. A fração coloidal do solo carrega uma carga negativa, o que normalmente repele os ânions. No entanto, a sorção de ânions nos solos, ocorre de forma particular e sob condições ácidas.

Existem algumas evidências de que a sorção do iodo por óxidos de ferro e alumínio ocorrem mais para iodato $\left(\mathrm{IO}_{3}^{-}\right)$do que para iodeto $\left(\mathrm{I}^{-}\right)$. Esses resultados apontam que essas propriedades são relevantes para explicar a concentração do iodo e seu background. No entanto, os efeitos na dinâmica do iodo, parecem mais associados ao efeito das condições físico-químicas do ambiente sobre esses componentes, associado às cargas elétricas em suas superfícies. Por essa razão, não serão considerados bons indicadores de fitodisponibilidade para a previsão de valores de $F v$.

\subsubsection{O pH}

$\mathrm{O}$ pH é um dos principais fatores que regula o comportamento e a biodisponibilidade dos radionuclídeos nos solos, pois interfere diretamente nos mecanismos de sorção e dessorção. Isso acontece porque muitas partículas inorgânicas e orgânicas do solo podem apresentar cargas elétricas nas suas superfícies dependentes do $\mathrm{pH}$. Em solos agrícolas das zonas tropicais, quando o pH está abaixo de 5,0, pode haver predominância de cargas positivas das partículas dos solos e consequentemente os ânions que estiverem em solução, serão fixados por estas partículas . À medida que o $\mathrm{pH}$ se eleva, com o uso de práticas, como a calagem por exemplo, passam a predominar as cargas negativas nas superfícies das partículas dos solos. Logo, os ânions que estavam antes sorvidos nestas superfícies retornam novamente para a solução do solo, pois estes sítios passam a atrair os cátions para a sorção. 
O iodo pode ser encontrado na natureza de várias formas químicas e o pH do solo pode influenciar nas espécies, influenciando no teor de iodo neste ecossistema . Shpeppard observou em seu experimento, que a maior sorção do iodo aconteceu em solos com pH baixo. Provavelmente, as espécies químicas presentes nestes solos estavam com cargas negativas, como o iodeto $\left(\mathrm{I}^{-}\right)$e o iodato (IO3-). Whitehead sugeriu que o pH baixo favorece a adsorção de iodo por óxidos de ferro e alumínio. Em contrapartida, os solos alcalinos, têm pouca capacidade de reter o iodo .Como se pôde depreender da discussão acima, o pH apresenta um papel preponderante no processo de fitodisponibilidade do iodo, explicando inclusive o efeito de minerais secundários na dinâmica do mesmo. Por essa razão, o pH será considerado como outra informação crucial para a previsão dos valores de $F v$ para o iodo.

\subsubsection{O Eh}

O iodo pode ser encontrado na natureza em vários estados de valência e numa gama de formas inorgânicas e orgânicas, incluindo iodeto $\left(\mathrm{I}^{-}\right)$, iodato $\left(\mathrm{IO}_{3}^{-}\right)$, iodo elementar $\left(\mathrm{I}_{2}\right)$ e iodo orgânico. As formas do iodo na natureza dependem em parte do $\mathrm{pH}$ e também do status de redução do ambiente circundante . Fuge e Johnson observaram que o iodo elementar $\left(\mathrm{I}_{2}\right)$ é oxidado em um meio alcalino, se transformando em iodato $\left(\mathrm{IO}_{3}{ }^{-}\right)$. Contudo, observou que os processos de dessorção do iodo em solos alagados não estavam atuando sozinhos. Outros processos podem estar atuando em condições de baixo Eh, já que os solos que continham a presença de matéria orgânica ou de plantas cultivadas neste ambiente, poderiam estar liberando exsudatos radiculares e por consequência, aumentando o estímulo de atividades microbianas; promovendo maior dessorção do iodo, quando comparado aos solos que também estavam em condições redutoras sem estes componentes. No entanto, o iodeto $\left(\mathrm{I}_{2}^{-}\right)$, o iodo elementar $\left(\mathrm{I}_{2}\right)$ e iodato $\left(\mathrm{IO}_{3}^{-}\right)$, são todos sujeitos a sorção por componentes dos solos.

De fato, observou-se que a concentração do iodo na solução do solo, aumenta em baixos valores de Eh obtidos após a irrigação nos meses de verão . Assim, além do potencial de redução química de $\mathrm{IO}_{3}^{-}$para $\mathrm{I}^{-}$, a anoxia aparentemente induz a dessorção, aumentando a concentração do iodo na solução do solo. Segundo Rowell , o pH de solos ácidos aumenta em torno da neutra- 
lidade como resultado da anoxia. Esse processo, por sua vez, leva a uma diminuição na carga líquida, negativa e dependente do $\mathrm{pH}$, associada aos sólidos do solo e com isso, espécies aniônicas sorvidas (carregadas negativamente) podem ser eletrostaticamente repelidas para a solução .

Assim, com base nesses comportamentos, entende-se que o Eh, por atuar na especiação do iodo, pode interferir no processo de fitodisponibilidade. No entanto, passa a ser um parâmetro relevante ao se comparar cultivos, como o do arroz, que podem ser realizados tanto em condições oxidantes, como em condições redutoras, sendo esta última, responsável por valores de $F v$ mais elevados . Portanto, considerando que as raízes da maioria das plantas só são capazes de sobreviver em solos com condições mais oxidantes e que o papel do $\mathrm{pH}$ explicaria a disponibilidade do iodo também em ambientes anóxicos, este estudo não vai considerar o Eh como um indicador crucial para a previsão de valores de Fv no sistema solo-planta.

\subsubsection{Os microrganismos}

Os microrganismos presentes nos solos possuem a capacidade de acumular o iodo, atuando de forma importante nos processos de sorção e dessorção deste elemento nos solos . Com o objetivo de entender a participação microbiana nos processos de acumulação do iodo em solos, Muramatsu realizou experimentos com radioiodo em amostras de solos, onde parte deste material, foi submetido a esterilização em autoclave. Em análise as espécies químicas do iodo presentes nestas amostras, observou-se que tanto $\mathrm{I}^{-} \mathrm{e} \mathrm{IO}_{3}{ }^{-}$foram sorvidos nos solos. No entanto, parte destas amostras foram esterilizadas em autoclave, apresentando uma redução drástica no processo de adsorção do iodo. Provavelmente esta redução ocorreu em função da destruição dos microrganismos que fixam este elemento no solo, ou pela decomposição de enzimas produzidas por estes indivíduos, que também atuam neste processo .

Alguns autores realizaram experimentos com a fumigação em solos (aplicação de pesticidas ou compostos químicos), com o objetivo de verificar se a presença dos microrganismos influencia na sorção e dessorção do iodo neste ambiente, e também encontraram uma redução deste elemento após a submissão a este tratamento . Através da revisão realizada neste estudo, foram 
identificados 28 artigos, que apontam a relevância dos microrganismos no estudo do iodo em solos.

Com base nesses comportamentos, entende-se que os microrganismos, podem efetivamente interferir no processo de fitodisponibilidade. No entanto, este estudo não vai considerar os microrganismos como um indicador crucial para a previsão de valores de $F v$ no sistema solo-planta, pois, além de serem de difícil avaliação em termos de diversidade e quantidade, os mesmos atuam sobre a matéria orgânica do solo, e, portanto, indiretamente, podem ser avaliados por seu teor no solo.

\subsection{Valores de $F v$ compilados}

Nesta revisão, foram levantados 363 valores de $F v$ para o iodo na literatura que atenderam aos critérios estabelecidos neste estudo. Assim, a partir desse levantamento, considerando os indicadores de fitoabsorção detectados para o iodo, o pH e o teor de matéria orgânica dos solos subjacentes, somente 135 valores continham essas informações. Assim, somente dois "learning sets" foram obtidos com um número mínimo para o treinamento através do uso de rede neural artificial: um para trigo (Tabela 4) e outro para alface (Tabela 5). A Tabela 5, embora não tenha o teor de matéria orgânica, apresenta o teor de húmus dos solos, além de várias outras propriedades pedológicas. Esses valores, apresentam restrições que podem inviabilizar sua aplicação global, pois foram obtidos de um único artigo, um mesmo país ou região, mesma condição climática e com pequena amplitude de valores de $\mathrm{pH}$ e matéria orgânica.

Tabela 4: Valores tabelados para a aprendizagem por rede neural artificial para fins de previsão de valores de $F v$ do ${ }^{125}$ I para trigo, tendo por base as propriedades dos solos [16].

\begin{tabular}{cccc}
\hline Fv para trigo & $\boldsymbol{p H}$ & $\begin{array}{c}\text { \% Carbono } \\
\text { Orgânico }\end{array}$ & \% Argila \\
\hline $1,20 \mathrm{E}-03$ & 5,4 & 1,94 & 31 \\
$3,70 \mathrm{E}-03$ & 5,5 & 1,44 & 13
\end{tabular}




\begin{tabular}{|c|c|c|c|}
\hline $1,20 \mathrm{E}-03$ & 5,6 & 1,92 & 17 \\
\hline $1,80 \mathrm{E}-03$ & 5,7 & 1,63 & 22 \\
\hline $2,40 \mathrm{E}-03$ & 5,7 & 1,63 & 12 \\
\hline $6,30 \mathrm{E}-04$ & 5,8 & 1,62 & 22 \\
\hline $1,30 \mathrm{E}-03$ & 5,8 & 1,74 & 23 \\
\hline $3,00 \mathrm{E}-03$ & 5,8 & 1,31 & 18 \\
\hline $1,70 \mathrm{E}-02$ & 5,8 & 1,31 & 15 \\
\hline $1,20 \mathrm{E}-03$ & 5,9 & 1,35 & 23 \\
\hline $1,50 \mathrm{E}-03$ & 6,0 & 1,78 & 38 \\
\hline $6,00 \mathrm{E}-03$ & 6,1 & 1,67 & 12 \\
\hline $1,00 \mathrm{E}-02$ & 6,1 & 2,41 & 6 \\
\hline $1,10 \mathrm{E}-03$ & 6,3 & 2,21 & 70 \\
\hline $2,50 \mathrm{E}-03$ & 6,3 & 1,55 & 14 \\
\hline $2,80 \mathrm{E}-03$ & 6,4 & 2,43 & 17 \\
\hline $3,70 \mathrm{E}-03$ & 6,4 & 1,36 & 21 \\
\hline $3,10 \mathrm{E}-03$ & 6,8 & 1,72 & 21 \\
\hline 1,00E-03 & 6,9 & 1,94 & 23 \\
\hline $3,60 \mathrm{E}-03$ & 6,9 & 1,33 & 14 \\
\hline 9,90E-03 & 7,1 & 1,84 & 17 \\
\hline $1,60 \mathrm{E}-03$ & 7,2 & 2,1 & 15 \\
\hline $2,00 \mathrm{E}-03$ & 7,3 & 1,61 & 18 \\
\hline $1,50 \mathrm{E}-03$ & 7,3 & 1,34 & 15 \\
\hline $9,20 \mathrm{E}-04$ & 7,3 & 2,58 & 14 \\
\hline $1,60 \mathrm{E}-03$ & 7,4 & 2,12 & 21 \\
\hline $2,10 \mathrm{E}-03$ & 7,4 & 2,03 & 16 \\
\hline $8,20 \mathrm{E}-04$ & 7,5 & 2,20 & 25 \\
\hline $8,10 \mathrm{E}-03$ & 7,5 & 1,93 & 26 \\
\hline 1,00E-03 & 7,5 & 2,11 & 32 \\
\hline $6,60 \mathrm{E}-04$ & 7,5 & 2,2 & 28 \\
\hline $9,50 \mathrm{E}-04$ & 7,5 & 2,52 & 25 \\
\hline $5,30 \mathrm{E}-04$ & 7,5 & 2,2 & 31 \\
\hline $1,70 \mathrm{E}-03$ & 7,5 & 1,73 & 14 \\
\hline $3,00 \mathrm{E}-03$ & 7,5 & 1,25 & 17 \\
\hline $1,20 \mathrm{E}-03$ & 7,6 & 3,42 & 18 \\
\hline $1,10 \mathrm{E}-03$ & 7,6 & 2,28 & 25 \\
\hline $4,70 \mathrm{E}-04$ & 7,6 & 2,15 & 35 \\
\hline
\end{tabular}


Tabela 5: Valores tabelados para a aprendizagem por rede neural artificial para fins de previsão de valores de $F v$ do ${ }^{125} \mathrm{I}$ para alface, tendo por base propriedades dos solos [72].

\begin{tabular}{ccccccc}
\hline $\begin{array}{c}\boldsymbol{F} \text { v para } \\
\text { alface }\end{array}$ & $\boldsymbol{p H}$ & $\begin{array}{c}\boldsymbol{p H} \\
\boldsymbol{e m} \mathbf{K C l}\end{array}$ & $\begin{array}{c}\boldsymbol{\%} \\
\boldsymbol{H u ́ m u s}\end{array}$ & $\boldsymbol{\%} \boldsymbol{N}$ & $\begin{array}{c}\boldsymbol{C a}(\mathbf{c m o l c} / \\
\mathbf{k g})\end{array}$ & $\boldsymbol{C T C}$ \\
\hline $4,00 \mathrm{E}-02$ & 6,3 & 5,2 & 0,8 & 0,06 & 1,6 & 5,8 \\
$5,00 \mathrm{E}-03$ & 7,9 & 6,9 & 1,1 & 0,06 & 5,9 & 14 \\
$3,00 \mathrm{E}-03$ & 7,9 & 6,9 & 1,1 & 0,06 & 5,9 & 14 \\
$3,60 \mathrm{E}-03$ & 7,9 & 6,9 & 1,1 & 0,06 & 5,9 & 14 \\
$2,70 \mathrm{E}-03$ & 7,9 & 6,9 & 1,1 & 0,06 & 5,9 & 14 \\
$6,00 \mathrm{E}-03$ & 8,2 & 7,00 & 2,8 & 0,18 & 12 & 26 \\
$8,00 \mathrm{E}-03$ & 8,2 & 7,00 & 2,8 & 0,18 & 12 & 26 \\
$4,10 \mathrm{E}-03$ & 8,2 & 7,00 & 2,8 & 0,18 & 12 & 26 \\
$7,00 \mathrm{E}-03$ & 8,2 & 7,00 & 2,8 & 0,18 & 12 & 26 \\
$1,10 \mathrm{E}-03$ & 8,2 & 7,00 & 2,8 & 0,18 & 12 & 26 \\
$4,00 \mathrm{E}-03$ & 8,2 & 7,00 & 2,8 & 0,18 & 12 & 26 \\
$1,30 \mathrm{E}-02$ & 8,6 & 7,3 & 4,0 & 0,27 & 22 & 35 \\
$7,00 \mathrm{E}-03$ & 8,6 & 7,3 & 4,0 & 0,27 & 22 & 35 \\
$1,60 \mathrm{E}-03$ & 8,6 & 7,3 & 4,0 & 0,27 & 22 & 35 \\
\hline
\end{tabular}

\section{CONCLUSÃO}

Este trabalho evidenciou que o comportamento do iodo no sistema solo-planta é bastante complexo, porém relativamente bem conhecido. A revisão bibliográfica sistemática apontou o pH e a matéria orgânica como propriedades cruciais para explicar os valores de $F v$ e portanto esses parâmetros foram selecionados como potenciais indicadores edafológicos para o iodo. De um modo geral, espera-se que solos ácidos e/ou com elevado teor de matéria orgânica apresentem baixa transferência do iodo para plantas. Solos tropicais, geralmente apresentam grau elevado de 
intemperismo e podem ser ricos em sesquióxidos de Fe a Al, apresentar baixo teor de matéria orgânica, e baixo valores de pH (solos ácidos). Nesses solos, por exemplo, espera-se que o iodo presente na solução sob a forma de ânions, seja fixado nas superfícies minerais dependentes do pH (sesquióxidos de Fe a Al), apresentando baixos valores de $F v$. No entanto, quase todos os valores compilados de $F v$ para o iodo, foram gerados em clima temperado ou em clima subtropical; e o uso destes valores como referência para gestão de acidentes em solos de clima tropical, poderá subestimar ou superestimar as doses pela a ingestão destes alimentos. Vale mencionar ainda, que solos tropicais podem ser submetidos à práticas agrícolas; como a calagem, que poderá promover mudanças no comportamento do iodo, reforçando a necessidade de se estabelecer um modelo conceitual global, o que só pode ser obtido estendendo estudos para ambientes tropicais, nos quais espera-se um comportamento diferenciado, mas essa tendência precisa ser validada.

No que se refere ao levantamento dos valores de $F v$ para o iodo, este trabalho identificou que a literatura ainda reporta os mesmos de maneira deficiente; seja através de informações pedológicas insuficientes ou ausentes, seja pela apresentação de valores reportados como médias ou em intervalos, apesar do princípio já bem estabelecido, na área da radioecologia, de que os valores de $F v$ variam em função das propriedades dos solos. A deficiência de valores de $F v$ para um mesmo isótopo do iodo, associados às propriedades dos solos, dificultaram a formação de uma base de dados robusta para o treinamento de uma rede neural, com o objetivo de prever valores de $F v$ a partir das propriedades dos solos. Desse modo, foi possível estruturar somente dois "learning set", um para trigo $(n=38)$ e outro para alface $(n=14)$, apesar das restrições que podem inviabilizar sua aplicação global, visto que foram oriundos de uma mesma região e portanto apresentam pequena amplitude de valores de pH e matéria orgânica. Estes learning sets são aceitáveis para se validar a suficiência dos indicadores selecionados para a previsão de valores de $F v$.

\section{AGRADECIMENTO}

Os autores agradecem à CNEN por uma bolsa de doutorado e à Capes por uma bolsa de pesquisa sênior (processo 88881.119173/2016-01). 


\section{REFERÊNCIAS}

[1].BELL, J. N.; SHAW, G. Ecological lessons from the Chernobyl accident. Environment international, v. 31, n. 6, p. 771-777, Aug 2005.

[2]. IAEA. Handbook of parameter values for the prediction of radionuclide transfer in terrestrial and freshwater environments. International Atomic Energy Agency. Vienna, 2010. p.208.

[3].FRISSEL, M. J. et al. Generic values for soil-to-plant transfer factors of radiocesium. Journal of Environmental Radioactivity, v. 58, n. 2-3, p. 113-128, 2002.

[4].VELASCO, H.; AYUB, J. J.; SANSONE, U. Influence of crop types and soil properties on radionuclide soil-to-plant transfer factors in tropical and subtropical environments. Journal of environmental radioactivity, v. 100, n. 9, p. 733-738, 2009.

[5]. SHEPPARD, S. C. et al. Revision and meta-analysis of selected biosphere parameter values for chlorine, iodine, neptunium, radium, radon and uranium. Journal of Environmental Radioactivity, v. 89, n. 2, p. 115-137, 2006.

[6]. BERESFORD, N. A. et al. Thirty years after the Chernobyl accident: What lessons have we learnt? Journal of Environmental Radioactivity, v. 157, p. 77-89, 2016.

[7]. MICHEL, R. et al. Retrospective dosimetry of Iodine-131 exposures using Iodine-129 and Caesium-137 inventories in soils - A critical evaluation of the consequences of the Chernobyl accident in parts of Northern Ukraine. Journal of Environmental Radioactivity, v. 150, p. 20-35, 2015.

[8].DARAOUI, A. et al. Iodine-129, Iodine-127 and Caesium-137 in the environment: soils from Germany and Chile. Journal of Environmental Radioactivity, v. 112, p. 8-22, 2012.

[9].RAO, U. S.; FEHN, U. Sources and reservoirs of anthropogenic iodine-129 in western New York. Geochimica Et Cosmochimica Acta, v. 63, n. 13-14, p. 1927-1938, 1999. 
[10]. JABBAR, T.; WALLNER, G.; STEIER, P. A review on 129I analysis in air. Journal of Environmental Radioactivity, v. 126, p. 45-54, 2013.

[11]. ALDAHAN, A.; ALFIMOV, V.; POSSNERT, G. I-129 anthropogenic budget: Major sources and sinks. Applied Geochemistry, v. 22, n. 3, p. 606-618, 2007.

[12].ZHANG, L. et al. Level and source of 129I of environmental samples in Xi'an region, China. Science of The Total Environment, v. 409, n. 19, p. 3780-3788, 2011.

[13].HU, Q.-H.; WENG, J.-Q.; WANG, J.-S. Sources of anthropogenic radionuclides in the environment: a review. Journal of Environmental Radioactivity, v. 101, n. 6, p. 426437, 2010.

[14]. KODAMA, S. et al. Speciation of iodine in solid environmental samples by iodine Kedge XANES: Application to soils and ferromanganese oxides. Science of the Total Environment, v. 363, n. 1-3, p. 275-284, 2006.

[15]. HOU, X. et al. A review on speciation of iodine-129 in the environmental and biological samples. Analytica Chimica Acta, v. 632, n. 2, p. 181-196, 2009.

[16].ZHU, Y. G. et al. Iodine uptake by spinach (Spinacia oleracea L.) plants grown in solution culture: effects of iodine species and solution concentrations. Environment International, v. 29, n. 1, p. 33-37, 2003.

[17]. SHINONAGA, T. et al. Transfer of iodine from soil to cereal grains in agricultural areas of Austria. Science of the Total Environment, v. 267, n. 1-3, p. 33-40, 2001.

[18]. BAN-NAI, T.; MURAMATSU, Y. Transfer factors of radioiodine from volcanic-ash soil (Andosol) to crops. J Radiat Res, v. 44, n. 1, p. 23-30, 2003.

[19]. SOUSA, M. R. D.; RIBEIRO, A. L. P. Revisão sistemática e meta-análise de estudos de diagnóstico e prognóstico: um tutorial. Arquivos Brasileiros de Cardiologia, v. 92, p. 241-251, 2009.

[20]. ASTAKHOVA, L. N. et al. Chernobyl-related thyroid cancer in children of Belarus: a case-control study. Radiat Res, v. 150, n. 3, p. 349-56, 1998. 
[21]. HUMPHREY, O. S. et al. Iodine soil dynamics and methods of measurement: a review. Environmental Science-Processes \& Impacts, v. 20, n. 2, p. 288-310, 2018.

[22]. FARIDULLAH et al. Socio-demographic characters, distribution and transformation of iodine in soil, plant and wheat grains at District Diamer, Gilgit-Baltistan, Pakistan. Environmental Geochemistry and Health, v. 40, n. 2, p. 777-790, 2018.

[23]. YU, H. et al. The distribution characteristics of halogen elements in soil under the impacts of geographical backgrounds and human disturbances. Geoderma, v. 305, p. 236$249,2017$.

[24]. SODERLUND, M. et al. Sorption and speciation of iodine in boreal forest soil. Journal of Radioanalytical and Nuclear Chemistry, v. 311, n. 1, p. 549-564, 2017.

[25]. SMOLEN, S.; LEDWOZYW-SMOLEN, I.; SADY, W. Iodine biofortification of spinach by soil fertigation with additional application of humic and fulvic acids. New Zealand Journal of Crop and Horticultural Science, v. 45, n. 4, p. 233-250, 2017.

[26]. SANTSCHI, P. H. et al. Recent advances in the detection of specific natural organic compounds as carriers for radionuclides in soil and water environments, with examples of radioiodine and plutonium. Journal of Environmental Radioactivity, v. 171, p. 226233, 2017.

[27]. SANTSCHI, P. H. et al. Iodine and plutonium association with natural organic matter: A review of recent advances. Applied Geochemistry, v. 85, p. 121-127, 2017.

[28]. GONZALI, S.; KIFERLE, C.; PERATA, P. Iodine biofortification of crops: agronomic biofortification, metabolic engineering and iodine bioavailability. Current Opinion in Biotechnology, v. 44, p. 16-26, 2017.

[29]. GADZHIMUSIEVA, N. T.; SAIDIEVA, A. A. The Dynamics of Mobile Iodine Forms in Components of Arid Ecosystems of the Western Caspians. Arid Ecosystems, v. 7, n. 2, p. 125-129, 2017. 
[30]. ALMAHAYNI, T. et al. Effects of incubation time and filtration method on Kd of indigenous selenium and iodine in temperate soils. Journal of Environmental Radioactivity, v. 177, p. 84-90, 2017.

[31]. XU, C. et al. Role of natural organic matter on iodine and 239,240Pu distribution and mobility in environmental samples from the northwestern Fukushima Prefecture, Japan. Journal of Environmental Radioactivity, v. 153, p. 156-166, 2016.

[32]. XU, S. et al. Carbon, cesium and iodine isotopes in Japanese cedar leaves from Iwaki, Fukushima. Journal of Radioanalytical and Nuclear Chemistry, v. 310, n. 2, p. 927934, 2016.

[33]. WISZNIEWSKA, A. et al. Natural Organic Amendments for Improved Phytoremediation of Polluted Soils: A Review of Recent Progress. Pedosphere, v. 26, n. 1, p. 1-12, 2016.

[34]. SMOLEN, S. et al. Biofortification of Carrot (Daucus carota L.) with Iodine and Selenium in a Field Experiment. Frontiers in Plant Science, v. 7, 2016.

[35]. SMOLEN, S. et al. The quality of carrot (Daucus carota L.) cultivated in the field depending on iodine and selenium fertilization. Folia Horticulturae, v. 28, n. 2, p. 151$164,2016$.

[36]. SMOLEN, S. et al. Iodine and selenium biofortification of lettuce (Lactuca sativa L.) by soil fertilization with various compounds of these elements. Acta Scientiarum Polonorum-Hortorum Cultus, v. 15, n. 5, p. 69-91, 2016.

[37]. SMOLEN, S.; LEDWOZYW-SMOLEN, I.; SADY, W. The role of exogenous humic and fulvic acids in iodine biofortification in spinach (Spinacia oleracea L.). Plant and Soil, v. 402, n. 1-2, p. 129-143, 2016.

[38]. DUBORSKA, E. et al. Aging and Substrate Type Effects on Iodide and Iodate Accumulation by Barley (Hordeum vulgare L.). Water Air and Soil Pollution, v. 227, n. 11, 2016.

[39]. DUBORSKA, E.; KUBOVA, J.; MATUS, P. Factors Affecting Iodine Mobility in Soils. Chemicke Listy, v. 110, n. 9, p. 625-629, 2016. 
[40]. TAKEDA, A. et al. Effect of aging on availability of iodine in grassland soil collected in Rokkasho, Japan. Journal of Radioanalytical and Nuclear Chemistry, v. 303, n. 2, p. 1191-1195, 2015.

[41]. MILLER, A. et al. Iodide uptake by negatively charged clay interlayers? Journal of Environmental Radioactivity, v. 147, p. 108-114, 2015.

[42]. TAKEDA, A. et al. Changes in the chemical form of exogenous iodine in forest soils and their extracts. Radiat Prot Dosimetry, v. 167, n. 1-3, p. 181-6, 2015.

[43]. YAMASAKI, S. et al. Bromine and iodine in Japanese soils determined with polarizing energy dispersive X-ray fluorescence spectrometry. Soil Science and Plant Nutrition, v. 61, n. 5, p. 751-760, 2015.

[44]. SIASOU, E.; WILLEY, N. Inter-Taxa Differences in Iodine Uptake by Plants: Implications for Food Quality and Contamination. Agronomy-Basel, v. 5, n. 4, p. 537-554, 2015 .

[45]. LUSA, M. et al. Sorption of radioiodide in an acidic, nutrient-poor boreal bog: insights into the microbial impact. Journal of Environmental Radioactivity, v. 143, p. 110-122, 2015 .

[46]. LINHARES, D. P. S. et al. Iodine environmental availability and human intake in oceanic islands: Azores as a case-study. Science of the Total Environment, v. 538, p. 531-538, 2015.

[47]. FUGE, R.; JOHNSON, C. C. Iodine and human health, the role of environmental geochemistry and diet, a review. Applied Geochemistry, v. 63, p. 282-302, 2015.

[48]. COX, E. M.; ARAI, Y. Environmental Chemistry and Toxicology of Iodine. In: SPARKS, D. L. (Ed.). Advances in Agronomy, v. 128, p.47-96, 2014.

[49]. CHANG, H.-S. et al. Model of radioiodine speciation and partitioning in organic-rich and organic-poor soils from the Savannah River Site. Journal of Environmental Chemical Engineering, v. 2, n. 3, p. 1321-1330, 2014. 
[50]. HURTEVENT, P. et al. Translocation of $125 \mathrm{I}, 75 \mathrm{Se}$ and $36 \mathrm{Cl}$ to Wheat edible parts following wet foliar contamination under field conditions. Journal of Environmental Radioactivity, v. 121, p. 43-54, 2013.

[51]. WENG, H. X. et al. Biogeochemical transport of iodine and its quantitative model. Science China-Earth Sciences, v. 56, n. 9, p. 1599-1606, 2013.

[52]. LUO, M. et al. Speciation and migration of 129I in soil profiles. Journal of Environmental Radioactivity, v. 118, p. 30-39, 2013.

[53]. CHOUNG, S. et al. Uptake Mechanism for Iodine Species to Black Carbon. Environmental Science \& Technology, v. 47, n. 18, p. 10349-10355, 2013.

[54]. BOWLEY, H. E. et al. Historical trends in iodine and selenium in soil and herbage at the Park Grass Experiment, Rothamsted Research, UK. Soil Use and Management, v. 33, n. 2, p. 252-262, 2017.

[55]. OLIVER, M. A.; GREGORY, P. Soil, food security and human health: a review. European Journal of Soil Science, v. 66, n. 2, p. 257-276, 2015.

[56]. KOROBOVA, E. M. et al. Iodine and Selenium Speciation in Natural Waters and Their Concentrating at Landscape-Geochemical Barriers. Geochemistry International, v. 52, n. 6, p. 500-514, 2014.

[57]. ASHWORTH, D. J. Transfers of Iodine in the Soil-Plant-Air System: Solid-Liquid Partitioning, Migration, Plant Uptake and Volatilization A2 - Preedy, Edited byVictor R. In: BURROW, G. N. e WATSON, R. (Ed.). Comprehensive Handbook of Iodine. San Diego: Academic Press, 2009. p.107-118.

[58]. SCHWEHR, K. A. et al. Organo-Iodine Formation in Soils and Aquifer Sediments at Ambient Concentrations. Environmental Science \& Technology, v. 43, n. 19, p. 72587264, 2009.

[59]. YAMADA, H. et al. Speciation of iodine in soils. Soil Science and Plant Nutrition, v. 45, n. 3, p. 563-568, 1999.

[60]. MEURER, E. J. Fundamentos de química do solo. Porto Alegre: Evangraf, 2006. 
[61]. FUGE, R.; JOHNSON, C. C. The geochemistry of iodine - a review. Environmental Geochemistry and Health, v. 8, n. 2, p. 31-54, 1986.

[62]. JOHANSON, K. J. Iodine in soil. Stockholm, Sweden: Swedish Nuclear Fuel and Waste Management Companyp, 2000. 45p. (SKB-TR--00-21).

[63]. WHITEHEAD, D. C. The distribution and transformations of iodine in the environment. Environment International, v. 10, n. 4, p. 321-339, 1984.

[64]. SHETAYA, W. H. et al. Iodine dynamics in soils. Geochimica Et Cosmochimica Acta, v. 77, p. 457-473, 2012.

[65]. YUITA, K.; KIHOU, N. Behavior of iodine in a forest plot, an upland field, and a paddy field in the upland area of Tsukuba, Japan: Vertical distribution of iodine in soil horizons and layers to a depth of 50 m. Soil Science and Plant Nutrition, v. 51, n. 4, p. 455-467, 2005.

[66]. ROWELL, D. L. Soil science: methods and applications. Harlow, UK: Longman Scientific \& Technical, 1994.

[67]. KOROBOVA, E. Soil and landscape geochemical factors which contribute to iodine spatial distribution in the main environmental components and food chain in the central Russian plain. Journal of Geochemical Exploration, v. 107, n. 2, p. 180-192, 2010.

[68]. MURAMATSU, Y.; YOSHIDA, S. Effects of microorganisms on the fate of iodine in the soil environment. Geomicrobiology Journal, v. 16, n. 1, p. 85-93, 1999.

[69]. MURAMATSU, Y. et al. Studies with natural and anthropogenic iodine isotopes: iodine distribution and cycling in the global environment. Journal of Environmental Radioactivity, v. 74, n. 1-3, p. 221-232, 2004.

[70]. KASHPAROV, V. et al. Soil-to-plant halogens transfer studies 1. Root uptake of radioiodine by plants. Journal of Environmental Radioactivity, v. 79, n. 2, p. 187-204, 2005 . 